\title{
Acidentes por animais peçonhentos no município de Guaraí (TO) no período de 2015-2017
}

O estudo com animais peçonhentos tem se tornado cada vez mais frequente devido ao alto número de acidentes nas áreas urbanas. Este trabalho tem o objetivo de avaliar os casos notificados por animais peçonhentos no município de Guaraí (TO) buscando caracterizar os fatores que influenciaram a incidência e prevalência dos acidentes na região. Trata-se de um estudo epidemiológico, descritivo. Utilizou-se os dados fornecidos pela Secretaria Municipal de Saúde/Vigilância Epidemiológica para o estudo temporal (2015-2017) na população investigada. As notificações dos acidentes com animais peçonhentos na região de Guaraí (TO) entre os anos de 2015 a 2017 totalizaram 170 casos, dos quais 62 não foram identificados. Por falta da Busca de assistência em saúde nessa região, é possível que ainda persista grande número de casos não notificados. $O$ ano de 2015 foi o ano de maior acidente com animais peçonhentos prevalecendo dentre os animais identificados às serpentes e em seguida os escorpiões. A presente análise mostrou que os acidentes por animais peçonhentos sofrem influência das atividades econômicas, culturais e ambientais.

Palavras-chave: Animais Peçonhentos; Intoxicação; Guaraí; Epidemiologia.

\section{Accidents by venomous animals in the municipality of Guaraí (TO) in the period 2015-2017}

\begin{abstract}
The study with animal animals has become more and more frequent due to the high number of accidents in urban areas. This work aims to evaluate the cases reported by the animals in Guarai (TO), seeking to characterize the factors that influenced the incidence and prevalence of accidents in the region. This is an epidemiological, descriptive study. The data provided by the MUNICIPAL secretariat of Health/epidemiological surveillance for the Temporal Study (2015-2017) were used in the investigated population. The notifications of accidents involving animals in the Guarai (TO) region between the years 2015 to 2017 totaled 170 cases, of which 62 were not identified. Due to the lack of the search for health care in this region, it is possible that many unreported cases persist. The year 2015 was the year of greatest accident with venomous animals prevailing among the animals identified to the snakes and then the Scorpions. The present analysis showed that accidents by farted animals are influenced by economic, cultural and environmental activities.
\end{abstract}

Keywords: Poison Animals; Intoxication; Guaraí; Epidemiology.

Topic: Epidemiologia

Reviewed anonymously in the process of blind peer.
Received: 11/12/2018

Approved: 15/01/2019
Eline Silva Alencar

Faculdade Guaraí, Brasil

http://lattes.cnpq.br/7442831139032218

eliny.2010@gmail.com

Maria Hananda Silva Araújo

Faculdade Guaraí, Brasil

hananda2014@gmail.com

Aluísio Vasconcelos de Carvalho (iD)

Faculdade Guaraí, Brasil

http://lattes.cnpq.br/5200758055263996

http://orcid.org/0000-0002-3793-3133

aluisiovasconcelos@gmail.com

\section{Referencing this:}

ALENCAR, E. S.; ARAÚJO, M. H. S.; CARVALHO, A. V.. Acidentes por animais peçonhentos no município de Guaraí (TO) no período de 2015-2017. Medicus, v.1, n.1, p.10-21, 2019. DOI: http://doi.org/10.6008/CBPC2674-6484.2019.001.0002 


\section{INTRODUÇÃO}

Animais peçonhentos são aqueles que inoculam substâncias tóxicas produzidas em glândulas específicas e tem a presença de um aparelho para inserir a sua peçonha na sua presa (COTTA, 2014). Acidentes envolvendo animais peçonhentos tem sido um dos maiores causadores de envenenamento humano no Brasil, perdendo apenas pela intoxicação por medicação (LEOBAS et al. 2016). Os incidentes com animais peçonhentos têm sido cada vez mais comuns nas zonas rurais nos países pobres como a África, Ásia, América Latina e Oceania (LADEIRA et al. 2017).

No mundo, são catalogadas 2.900 espécies de serpentes, divididas em 465 gêneros e 20 famílias. A fauna brasileira possui 321 espécies, 75 gêneros e 9 famílias (COTTA, 2014). No Brasil, segundo o Ministério da Saúde, ocorre em média 25 mil acidentes por ano, respondendo 87\% dos casos o gênero Bothrops (jararaca), Crotalus (cascavel) 9\%, Lachesis (surucucu) 3\% e Micrurus (cobra coral) representando cerca de 1 $\%$ dos casos (SINAN, 2013).

A ordem Scorpiones compreende 1.500 gêneros, com uma extensa distribuição geográfica, as espécies identificadas estão em todos os continentes, com exceção da Antártida. Estas espécies são reconhecidas em todas as regiões tropicais do mundo (COTTA, 2014). No Brasil, os escorpiões de importância médica faz parte do gênero Tityus, as espécies $T$. serrulatus (escorpião amarelo) com incidência na Bahia, Minas Gerais, São Paulo, Espírito Santo, Paraná, Rio de Janeiro e Goiás; T. bahiensis (escorpião marrom), em Goiás, São Paulo, Mato Grosso do Sul, Minas Gerais, Paraná, Santa Catarina e Rio Grande do Sul e T. stigmurus que ocorre nos estados da região Nordeste (COTTA, 2014).

A ordem Lepidoptera é uma das maiores ordens do Reino Animal, Cerca de 150.000 espécies já foram classificadas no planeta, no Brasil, 3.400 espécies (MORAIS, 2009; KERPEL et al. 2014). As larvas e os adultos são propícios a provocar acidentes. Os envenenamentos com lagartas e pupas de lepidópteras são representados como Erucismo. O envenenamento é anormal, mesmo não apresentando risco de vida para o indivíduo, com exceção da espécie e do acidentado, pode apresentar lesões dermatológicas leves, e em alguns casos, hematomas e convulsões (HADDAD JR et al. 2009).

A ordem Hymenoptera, compreende os animais que têm ferrões verdadeiros, e existem três famílias de interesse médico: Apidae (abelhas e mamangavas), Vespidae (vespa amarela, vespão e marimbondo ou caba) e Formicidae (formigas) (BRASIL, 2010). Proveniente de uma estrutura ovopositora alterada, somente as fêmeas têm a capacidade de ferroar. São muito perigosos, sendo capaz de provocar acidentes graves e até a morte seja por reações anafiláticas resultante de uma picada ou por intoxicação resultante de várias picadas (ALVES et al. 2017).

Os animais sinantrópicos, espécies indesejadas que convivem com seres humanos, tem se tornado cada vez mais prevalentes nas regiões tropicais, sobretudo em áreas urbanas, contribuindo para o alto número de acidentes com animais peçonhentos. $\mathrm{O}$ estudo da biologia do comportamento dessas espécies e a epidemiologia dos acidentes ocasionados por animais peçonhentos são necessários para desenvolver métodos eficazes de controle e prevenção de acordo com cada região. 
Este trabalho tem o objetivo de avaliar os casos notificados por animais peçonhentos no município de Guaraí (TO) buscando caracterizar os fatores que influenciaram a incidência e prevalência dos acidentes no município.

\section{METODOLOGIA}

Trata-se de um estudo epidemiológico, descritivo de caráter quantitativo. Utilizaram-se os dados fornecidos pela Secretaria municipal de Saúde/Vigilância Epidemiológica para o estudo temporal (2015-2017) na população investigada. Utilizou-se a estatística descritiva para análise dos dados de acordo com a faixa etária, tipo de acidente, zona do acidente e sexo.

De acordo com IBGE (2016), o município de Guaraí, área escolhida para o estudo, possui uma extensão territorial de 2.268 km 2 e está situado na Mesorregião Ocidental do Tocantins e Microrregião de Miracema do Tocantins, sede da 6a Região Administrativa do Estado, distante $173 \mathrm{~km}$ da capital do Estado (Palmas). Segundo o Instituto Brasileiro de Geografia e estatística - IBGE, possui uma população de 23.200 habitantes (censo demográfico de 2010) (Figura 1).

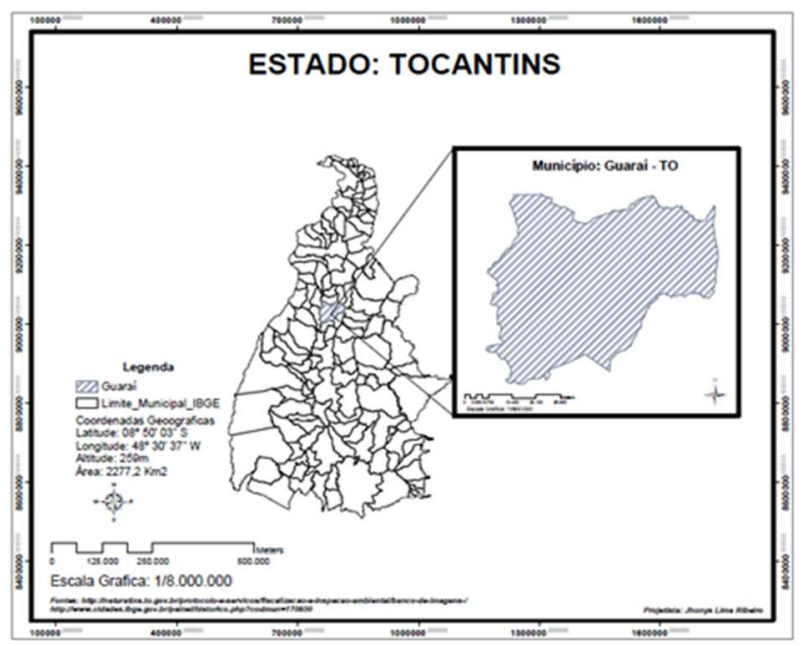

Figura 1: Mapa de Guaraí (TO). Fonte: IBGE.

\section{RESULTADOS E DISCUSSÃO}

As notificações dos acidentes com animais peçonhentos na região da superintendência da saúde de Guaraí (TO), entre os anos de 2015 a 2017 totalizaram 170 casos, dos quais 62 não foram identificados. Por falta da Busca de assistência em saúde nessa região, é possível que ainda persista grande número de casos não notificados.

O ano de 2015 representou o maior quantitativo de acidentes com animais peçonhentos com 72 casos e, 2016 e 2017 ocorreram 49 casos notificados prevalecendo dentre os animais identificados às serpentes e escorpiões (Tabela 1).

Os problemas relacionados a estes agravos são diversos, como uma leve queimadura, e até mesmo a morte do indivíduo em questão de pouco tempo (AZEVEDO, 2006). A complicação do acidente deve ao tipo de animal, quantia de substância tóxica injetada, imunidade do atacado, a quantidade de tempo entre o ataque e o socorro, a ausência de socorro básico de saúde, ou pela avaliação errada (CARDOSO et al. 2009). 
Tabela 01: Notificação por tipo de acidente. Fonte: Secretaria Municipal de Saúde.

\begin{tabular}{c|c|c|c|c|c|c|c}
\hline $\begin{array}{c}\text { Ano da } \\
\text { Notificação }\end{array}$ & Serpente & Aranha & Escorpião & Lagarta & Abelha & Outros & total \\
\hline 2015 & 15 & 0 & 12 & 9 & 13 & 23 & 72 \\
\hline 2016 & 13 & 2 & 5 & 2 & 6 & 21 & 49 \\
\hline 2017 & 9 & 5 & 11 & 2 & 4 & 18 & 49 \\
\hline Total & $\mathbf{3 7}$ & $\mathbf{7}$ & $\mathbf{2 8}$ & $\mathbf{1 3}$ & $\mathbf{2 3}$ & $\mathbf{6 2}$ & $\mathbf{1 7 0}$ \\
\hline
\end{tabular}

Há também aqueles que a população considera como acidente pouco importante, que não necessita de atendimento médico, mas com cuidados oriundos do conhecimento popular (ROSA et al. 2015). No Brasil no ano de 2010 a 2016, notificou-se 895.533 ataques por animais peçonhentos, 32\% dos casos encontravamse no Nordeste, $2 \%$ na Paraíba e $1 \%$ na Região Metropolitana de Campinas Grande-Paraíba (BARBOSA et al. 2012).

O CEATOX (Centro de Assistência Toxicológica) notifica os agravos dos ataques, relata os dados sociais do indivíduo, o gênero que o animal pertence, local da picada, diagnóstico do acidentado, entre outras informações (PARAÍBA, 2015). Para catalogação dessas informações são utilizados formulários de análise padronizados, após a coleta destas informações, o sistema nacional de informação sobre estes acidentes é atualizado.

Este material é de suma importância, carrega informação que pode ser utilizada na capacitação dos agentes de saúde e instituições, e até mesmo em relação ao acompanhamento dos acidentados. Mesmo assim, a coleta e as notificações desses acidentes têm erros, deixando lacunas dos aspectos biológicos que interfere na qualidade numérica destas informações. Tal fato explica o enorme número de notificações de acidentes por animais peçonhentos sem a identificação por parte do hospital e parte da população (figura 1). Outros animais, por não estarem catalogados na ficha de identificação, como o potó, lacraias, formigas, entram para o número exacerbado de notificações sem identificação.

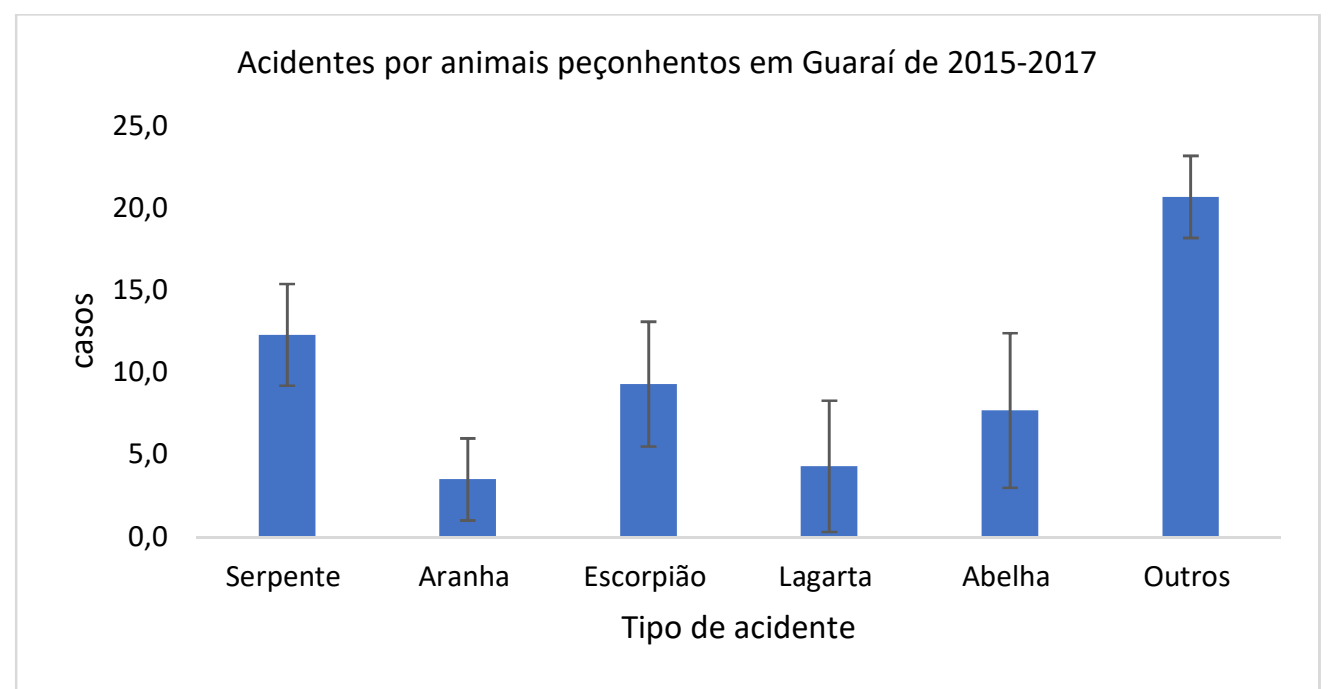

Figura 1: Média e Desvio-padrão de acidentes por animais peçonhentos em Guaraí (TO).

Os besouros (potós) são coleópteros vesicantes, os ataques causados por estes animais têm sido referidos em todos os continentes, exceto nas zonas polares. A maioria das espécies fazem parte do gênero Paederus (BRASIL, 2002). São populares em todo mundo, contém aproximadamente 600 espécies de 
Paederus, dividida pelas zonas tropicais e temperadas do globo. Cerca de $4 \%$ das espécies catalogadas desse gênero são identificadas como autora de dermatite linear (FRANK et al. 1987).

O tema questionado não se mostra como atualidade, uma vez que a primeira informação de ataque por Paederus (potós) data de 1912. Porém, nos últimos 15 anos vem dando um maior destaque, ainda assim de maneira preliminar e sem a importância necessária, pois, tais animais, em certo período do ano em algumas cidades do Nordeste ocasiona centenas de dermatites e sendo definidos como epidemias ou surtos (NEIVA et al. 1916; ALBUQUERQUE et al. 2000).

No Brasil são conhecidos por potó dois coleópteros distintos, todos os dois causam dermatite vesiculosa, também conhecida como dermatite linear, o Potó-pimenta e Potó grande ou Potó burrinho. São animais que fazem parte da família Meloidae; tem cerca de 1,5 a 3cm com dois gêneros - Lytta e Epicauta. Alimentam-se de leguminosas e hortaliças, batata, tomate, milho, dentre outros (ALBUQUERQUE et al., 2008).

O veneno produzido por estes insetos é a cantaridina (para o gênero Lytta e Epicauta), forma um forte incômodo e queimaduras (CARDOSO et al. 2003). A matéria vesicante fabricada pelos Paederus é a pederina, uma amida cristalina de forte atuação corrosiva e vesicante, solúvel em álcool e água, apto a impedir a mitoses atuando no DNA da célula. A vesicante tem diferença em comparação a cantaridina, causando eritema mais forte e vesículas de tamanho pequeno. Os sintomas de ardência e coceira são mais fortes nos ataques em que pederina está envolvida (FAIN, 1996).

Os animais da família Scolopendridea são identificados por diferentes nomes, piolho de cobra, lacraia ou centopeia. Predador de prática noturna, que se abriga em locais úmidos e escuros em meio às plantas e brechas das rochas, pode chegar até $25 \mathrm{~cm}$ de comprimento. Estes Myriapodas são classe Chilopoda, que se define por animais com um par de patas por segmentação, um par de antenas, ocelos laterais (gênero Ostostigmus), um para de olhos simples (gênero Scolopendra) (BARROSO et al. 2001), na última divisão há um par de apêndices anais e no abdome aparelho genital. Entre outras particularidades são Trignatos, apresentam dois pares de maxilas e um par de mandíbulas chamada forcípulas (BARBOSA et al. 2016).

As forcípulas são associação a glândulas de veneno que fabrica substância de impacto neurotóxico de baixa ação local e vasta ação sistêmica em mamíferos de pequeno porte (BÜCHERL, 1941). Há poucas informações de ataques informados, contudo os sintomas das ocorrências são de dor imediata e sensação de queimação forte persistente por 24 horas. Inchaço local e hiperemia podem ser examinada e transformar em inchaço maiores, necrose local e evoluir para febre, tremores, vômitos, cefaleia, dispneia e ansiedade (HIDAKA et al. 2001).

A quantidade de espécies de Chilopodas que tem no Brasil é incalculável. Segundo Kury et al., (2010) no Estado do Tocantins há 10 espécies e 9 morfotipos ainda não relatada. Na Amazônia, de acordo com Schileyko (2002) existem 53 espécies de lacraias, 40 delas são nativas do bioma. De acordo com Barroso, Hidaka et al. (2001), a espécie mais popular no país é a Scolopendra veridicornis. 
O gênero Solenopsis (Hymenoptera: Formicidae) compreende o grupo de formigas comumente identificada como "formigas lava-pés" ou "formigas de fogo". As espécies mais conhecidas no Brasil são Solenopsis saevissima Smith,1855 e Solenopsis invicta Buren, 1972 (BUENO et al. 1999), são duas espécies com vasta distribuição pelo Brasil (TRAGER, 1991; FOX, 2010), até mesmo na região urbana (ZERINGÓTA, 2014).

Estas formigas são conhecidas por seu ataque agressivo e pelos sintomas da picada gerando dor, queimação e coceira. No entanto, dependendo do número de picadas e a sensibilidade da pessoa, pode surgir inchaço no local da picada, pustulose, necrose de tecido, coceira por todo corpo e choque anafilático acarretando deficiência respiratória, e até a morte (DESHAZO, 1984; HADDAD, 2009). No Brasil o maior número de ataques de formigas está relacionado a espécie S. saevissima (MALASPINA, 2004).

Os acidentes com serpentes são mais prevalentes em determinadas épocas do ano. No Norte, Nordeste e Centro-Oeste, as serpentes podem ser encontradas ao longo de todo ano, mas preferem os tempos mais quentes, escondendo nas épocas mais frias (FREITAS, 2003). A redução de temperatura e ausência de chuvas ocasiona a queda do metabolismo das serpentes e influencia na diminuição das presas. O local mais frio e seco diminui a disponibilidade de caça mesmo em regiões tropicais ou subtropicais (MARQUES et al. 2009).

As serpentes manifestam suas atividades durante o ano diretamente associadas aos fatores climáticos (pluviosidade, temperatura e umidade), maiores recursos alimentares, limitações filogenéticas e procriação (HERNDERSON et al. 1978; MARQUES et al. 2000; OLIVEIRA et al. 2001; SILVA, 2017; BERNARDE et al. 2006; MARQUES et al. 2006; SILVA, 2017; PARPINELLI et al. 2008; TURCl et al. 2009).

O escorpião adapta a dependência urbanas, refugiando-se em ambiente com acúmulo de lixo, montes de tijolos, telhas e alimenta de insetos em geral. O desenvolvimento de moradias, a falta de coleta seletiva, o problema de saneamento básico e moradia com estrutura provisória, são fatores primordiais que favorecem a adaptação e sobrevivência desses animais na região habitacional (CANTER, 2008; NODARI et al., 2006).

Os ataques por escorpiões encontram-se agregado ao avanço desordenado da zona urbana, à impertinência de infraestrutura e a oscilação ambiental, que possibilita o aparecimento dos animais na cidade (ALBUQUERQUE et al. 2004; CANTER, 2008). O aumento da quantidade de lixo, de restos de alimento favorece o aumento da população de baratas, seu principal alimento, levando-os diretamente para as residências.

A capacitação de profissionais torna-se importante na identificação do animal e no tratamento correto para que haja um atendimento eficaz e melhores estratégias de divulgação de informação da prevenção, garantindo a redução de acidentes e de casos não identificados.

As pessoas possuem uma interação cultural com diversos tipos de animais (ULYSSES et al. 2010; SANTOS et al. 2015), entre eles temos as vespas sociais, identificada por marimbondo ou cabas e as abelhas (SOUZA et al. 2012). Esses insetos são vistos pelas pessoas com animais perigosos por causa da sua ferroada dolorosa que pode ocasionar sudorese, dor de cabeça, inchaço, desmaio e febre. 
O hábito do ataque é uma forma de defesa das colônias contra predadores, também é utilizada para diferenciar etnoespécies, no caso do exu-do-pasto, provavelmente umas espécies do gênero Brachygastra, termo normalmente usados em várias regiões do Brasil (SANTOS, 1995; SOUZA et al. 2008). Os padrões das picadas representam uma personalidade marcante para a definição popular das abelhas e maribondos seja no Brasil (COSTA-NETO, 2003) ou outros países como Honduras (OLTROGGE, 1975).

Os marimbondos (Hymenoptera: vespidae) identificada também por vespas, fazem parte de um grande grupo com diferentes espécies. Visto que de uma forma geral, tem uma poderosa peçonha com ação neuro e citotóxica, além de analgésica, hemolítica, hemorrágica e alergênica (MORTARI et al. 2005). A reação local provocada causa dor, edema e inchaço no local atingido, em geral são limitados. Uma grande quantidade de veneno injetado pode provocar reação tóxica sistêmica, provocando hemólise, coagulopatia, rabdomiólise, insuficiência renal aguda e hepatotoxicidade. No entanto, a maior parte das mortes está intimamente ligada a choque anafilático (EVANS et al. 1986; LORENZI, 2002).

Os Lepidópteras, representados pelas lagartas, possuem seis famílias de importância médica: Megalopygidae, Limacodidae, Saturniidae, Notordintidae, Arcitiidae e Limantriidae (KESSELRING et al. 1979; GUSMÃO et al. 2004; KERPEL et al. 2014). Os Lepidópteros possuem metamorfose completa, enquanto fase larval, os animais são herbívoros, isso faz com que estes animais fiquem em meio às árvores e arbustos facilitando o acidente ocasional embora os adultos (fase alada e reprodutiva) também provoque acidentes (MORAIS, 2009).

Os acidentes com aranhas ocorrem em maior número no outono, escorpiões e serpentes no verão e primavera, porém os insetos diminuem suas populações no inverno (SALOMAO, 2005). No período de chuva os animais peçonhentos são obrigados a acharem outro esconderijo tanto em zona urbana e rural não sendo difícil encontrá-los nas proximidades das casas, parques e jardins, o aumento desordenado da região urbana aumenta a exposição a estes acidentes.

Existem aproximadamente 35.000 espécies de aranhas no mundo, cerca de 30 espécies são consideradas perigosas para os humanos e pode causar intoxicação significativa. As espécies que causam acidentes de relevância médica no Brasil pertencem aos gêneros Phoneutria, Loxosceles e Latrodectus (INSTITUTO BUTANTAN, 2007).

Na maior parte das informações dos acidentes por espécies de aranhas não são notificados, tendo em vista que a maioria dos animais trazidos pelos atacados chegam deformados, podres ou incompleto devido a fúria do acidentado de matá-los e a fragilidade da sua estrutura, complicando o seu reconhecimento (LISE, 2006).

O araneísmo, além do interesse médico, é parcialmente negligenciado quando associada aos acidentes por animais peçonhentos (NÓBREGA, 2018). No entanto, ainda se caracteriza como complicação de saúde pública especialmente na zona tropical do planeta (CHAGAS, 2010). No Nordeste, anotações de ataque por aranhas são poucas e, provavelmente desvaloriza o verdadeiro perigo da situação (BRASIL, 2009).

A distribuição de acidentes por zona teve uma diferença significativa entre a zona urbana $(24,7 \pm 9,9)$ e zona rural $(32 \pm 3,6)$. Os acidentes no ano de 2015 a 2017, mesmo a área urbana não sendo um habitat 
natural, tem proporcionado um ambiente favorável a estes animais por meio da grande quantidade de lixo, entulhos e abrigo.

Os ataques por animais peçonhentos no Brasil vêm aumentando devido o desmatamento, a falta de higiene, saneamento básico e coleta seletiva (BRASIL, 2011; CARDOSO et al. 2013). É notável o aumento das notificações nos últimos anos nas residências mais próximas às áreas naturais, principalmente municípios com populações menores e rurais. Na Amazônia, o desmatamento é uma das causas devido a degradação do ambiente natural desses animais, faz com aconteça uma aproximação com a zona urbana (SACCARO JR et al. 2015).

Aranhas e escorpiões são um grande exemplo, pois tornam-se bastante adaptados à vida urbana e às condições proporcionadas pelas moradias humanas, que oferecem possibilidades de abrigo como lixos e entulhos, além de uma farta alimentação pela presença de baratas e outros insetos (BRASIL, 2001; LUCAS, 1988; SOARES et al., 2002).

$\mathrm{O}$ ataque de animais peçonhentos por sexo mostra uma grande diferença, o sexo masculino teve um índice maior $(44 \pm 11,8)$ que o feminino $(12,7 \pm 4,0)$ ao longo dos anos 2015 a 2017 . O estudo feito por Lima et al. (2009) encontrou uma taxa de 53,1\% em homens ao observar o perfil dos acidentes ofídicos no norte do estado de Minas Gerais. Da mesma maneira, Pinho et al. (2004) encontrou uma frequência de 78,5\% dos casos notificados no gênero masculino.

A população masculina possui um maior contato com o ambiente em que se encontram os animais peçonhentos, já que essa parcela está sujeita a atividades braçais aumentando o risco ao tipo trabalho. Esta explicação encontra suporte em Rojas et al. (2007) ao caracterizar o quadro dos acidentes ofídicos na região noroeste de São Paulo, o autor constatou que as circunstâncias dos eventos mostravam que 56\% dos agravos ocorreram durante atividade relacionada ao trabalho no campo.

A proporção de acidentes com indivíduos com a faixa etária de 20 aos 49 é maior (figura 2). Cardoso et al. (2007) ao estudar os acidentes no Estado de Santa Catarina identificaram uma maior incidência entre homens dos 20 aos 49 anos de idade, fato possivelmente relacionado ao contato destes indivíduos com situações de extremo risco, por exemplo, atividades no ramo da agricultura.

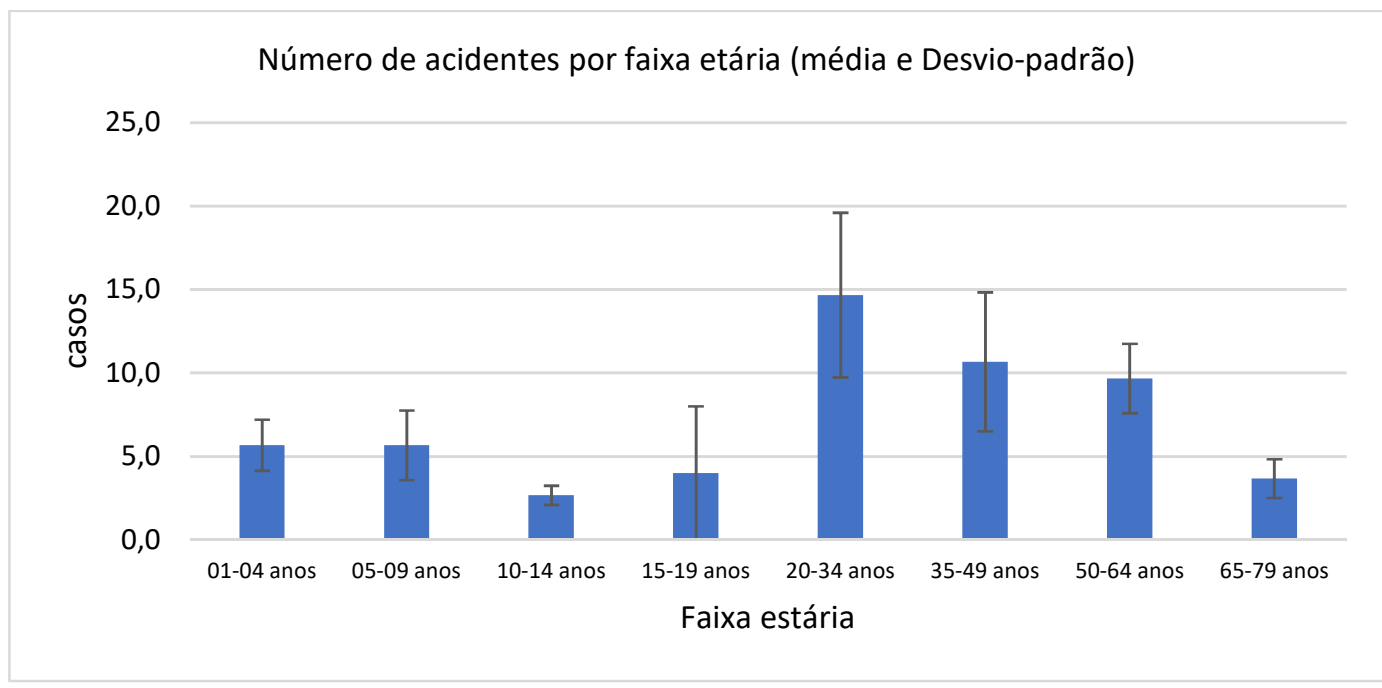

Figura 2. Média e desvio-padrão de acidentes por faixa etária no município de Guaraí (TO). 
As atividades agropecuárias sem a utilização dos equipamentos de proteção individual (EPI) possibilitam o risco a exposição aos animais peçonhentos, aumentando o índice de acidentes com esses animais (LIMA et al. 2006). Da mesma maneira, os trabalhos sem o uso de EPIs favorecem o elevado risco de acidentes com animais peçonhentos independentemente da zona, idade ou sexo.

No Tocantins e na maioria dos Estados brasileiros, as informações de acidentes com animais peçonhentos ainda são pouco estudadas do ponto de vista ecológico, é necessário pesquisa que possibilita uma visão abrangente do estado que são anormais na literatura para que o público tenha um conhecimento de forma clara e eficaz (LEOBAS et al. 2016).

Acidentes com animais peçonhentos é cada dia mais frequente, porém são casos negligenciados, seja pela falta de conhecimento hospitalar, laboratorial e por agentes qualificados na área para atender as vítimas e assim dando um socorro adequado segurado por lei (AZEVEDO, 2006; FISZON et al. 2008; ROSA et al. 2015).

\section{CONCLUSÃO}

A presente análise mostrou que os acidentes por animais peçonhentos ainda são pouco estudados, evidenciando sua relativa importância para a saúde pública, visto que teve muitos acidentes não reconhecidos tanto na zona urbana quanto na zona rural. Otimizar o sistema de notificação e identificação são cruciais para o efetivo programa de monitoramento de agravos à saúde do município.

Os resultados obtidos mostram que ainda há uma deficiência nos resultados da cidade de Guaraí, o número de resultados não identificados é muito grande. A importância de implementar estratégias de educação em saúde às comunidades atendidas, alertando as pessoas com as medidas preventivas, o uso dos EPIs, manutenção das residências limpas, influencia na redução dos acidentes por animais peçonhentos. Além disso, a capacitação de profissionais da saúde para a identificação da espécie contribui para o rápido diagnóstico e tratamento dos acidentados..

\section{REFERÊNCIAS}

ALBUQUERQUE, H. N.; LEITE, C. F.; ALBUQUERQUE, I. C. S.; CAVALCANTI, M. L. F.. Contribuição ao estudo dos potós (Paederus sp) em dois bairros da cidade de Campina GrandePB. BIOFAR: Rev Biol Far, v.3, n.1, p.26-37, 2008.

ALBUQUERQUE, H. N.; OLIVEIRA JÚNIOR, J. N.; SALES, I. C.. Acidentes por potós em escolares de uma escola da rede privada do bairro das Malvinas em Campina Grande Paraíba. In: SEMANA CAMPINENSE DE BIOLOGIA, 8. Anais. Campina Grande: 2000.

ALBUQUERQUE, I. C. S.; ALBUQUERQUE, H. N.; ALBUQUERQUE, E. F.; NOGUEIRA, A. S.; CAVALCANTI, M. L. F.. Escorpionismo em Campina Grande - PB. Rev Biol Ciênc Terra, v.4, n.1, 2004.

ALVES, F.; MACHADO, C.; LEMOS, E. R. S.. Formigas e vespas como problema de saúde pública. Journal Health Npeps, v.2, n.1, p.122-129, 2017.

AZEVEDO, J. L. S. D.. A importância dos centros de informação e assistência toxicológica e sua contribuição na minimização dos agravos à saúde e ao meio ambiente no Brasil. Dissertação (Mestrado em Desenvolvimento Sustentável) - Universidade de Brasília, Brasília, 2006.

BARBOSA, A. R.. Animais Peçonhentos: Notificação dos Espécimes. Revista Brasileira de Toxicologia, v.25, p.114, 2012.

BARBOSA, A. R.. Sinantrópicos peçonhentos: sistema de notificação de acidentes e considerações biológicas. Tese (Doutorado em Desenvolvimento e Meio Ambiente) Universidade Federal da Paraíba, João Pessoa, 2016.

BARROSO, E.; HIDAKA, A. S. V.; SANTOS, A. X.; FRANÇA, J. D. A. M.; SOUSA, A. M. B.; VALENTE, J. R.; PARDAL, P. P. O.. Acidentes por centopeia notificados pelo "Centro de informações Toxicológicas de Belém", num período de dois anos. Revista da Sociedade Brasileira de Medicina Tropical, v.6, n.34, p.527-530, 2001.

BARROSO, E.; HIDAKA, A. S. V.; SANTOS, A. X.; FRANÇA, J. D. M.; SOUZA, A. M. B.; VALENTE, J. R.; MAGALHÃES, A. F. A.; 
PARDAL, P. P. O.. Acidentes por centopeia notificados pelo "Centro de informações Toxicológicas de Belém", num período de dois anos. Revista da Sociedade Brasileira de Medicina Tropical, v.6, n.34, p.527-530, 2001.

BERNARDE, P. S.; ABE, A. S.. A snake community at Espigão do Oeste, Rondônia, Southwestern Amazon, Brasil. South American Journal of Herpetology, v.1, n.2, p.102-113, 2006

BRASIL, T. K.; PINTO-LEITE, C. M.; SILVA, L. M. A.; LIRA, R. M. S.; BRESCOVIT, A. D.. Aranhas de importância médica do Estado da Bahia, Brasil. Gazeta Médica da Bahia, v.79, n.1, 2009.

BRASIL. Acidentes por animais peçonhentos: Notificações registradas no Sistema de Informação de Agravos de Notificação. Brasília: SINAN-NET, 2017.

BRASIL. Ministério da Saúde. Fundação Nacional de Saúde. Manual de diagnóstico e tratamento de acidentes por animais peçonhentos. 2 ed. Brasília: Fundação Nacional de Saúde, 2001.

BRASIL. Ministério da Saúde. Fundação Oswaldo Cruz. Casos, Óbitos e Letalidade de Intoxicação Humana por Agente e por Região - Brasil. Brasília: SINITOX, 2010.

BRASIL. Ministério da Saúde. Manual de Diagnóstico e Tratamento de Acidentes por Animais Peçonhentos. Brasília: Fundação Nacional de Saúde, 2002.

BRASIL. Número de acidentes com animais peçonhentos dobra em dez anos. Brasília: Portal Brasil, 2011.

BÜCHERL, W.. Catálogo dos Quilópodes da Zona Neotropical. Memórias do Instituto Butantã, São Paulo, v.15, p.251-372, 1941.

BUENO, O. C.; CAMPOS-FARINHA, A. E.C.. As formigas domésticas. Insetos e outros invasores de residências. Piracicaba: FEALQ, 1999

CANTER, H. M.; KNYSAK, I.; CANDIDO, D. M.. Aranhas e escorpiões e lacraias. 2008.

CARDOSO, C. F. L.; SOARES, M. A.. Animais peçonhentos do município de Mangaratiba, RJ. Novo Enfoque, v.16, n.16, p.25-40. 2013.

CARDOSO, D. C.; CRISTIANO, M. P.; SANTOS, R. M.; COSTA S.; ZOCCHE, J. J.. Epidemiology and injuries (1994-2005) resulting from poisonous animals in southern Santa Catarina State, Brazil. Journal Public Health, v.15, n.6, p.467-472, 2007.

CARDOSO, J. L. C.; FRANÇA, F. O. S.; WEN, F. H.; MÁLAQUE, C. M. S.; HADDAD JUNIOR., V.. Animais peçonhentos do Brasil: biologia, clinica e terapêutica. São Paulo: Sarvier, 2009.

CHAGAS, F. B.; D'AGOSTONI, F. M.; BETRAME, V.. Aspectos epidemiológicos dos acidentes por aranhas no Estado do Rio Grande do Sul, Brasil. Evidência-Interdisciplinar, v.10, n.1-2, p.121-130, 2012.

COSTA NETO, E. M.. Etnoentomologia no povoado de Pedra Branca, município de Santa Terezinha, Bahia. Um estudo de caso das interações seres humanos/insetos. Tese (Doutorado) - Universidade Federal de São Carlos, São Carlos, 2003.

COTTA, G. A.. Animais peçonhentos. Belo Horizonte: Fundação Ezequiel Dias, 2014.

DESHAZO, R. D.; GRIFFING, C.; KWAN, T. H.; BANKS, W. A.; DVORAK, H. F.. Dermal hypersensitivity reactions to imported fire ants. J Allergy Clinical Immunology, v.74, n.6, p.841-847, 1984

EVANS, R.; SUMMERS, S.. Clinical aspects of Hymenoptera sensitivity. In: LEVINE, M.I.; LOCKEY, R. F.. American Academy of Allergy and Immunology. Pittsburgh: Lambert Associates, 1986

FAIN, A.. Toxic action of bove beetles (coleoptera, Staphylinidae). Memorias do Instituto Butantan, v.33, n.3, p.835-844, 1966

FISZON, J. T.; BOCHNER, R.. Subnotificação de acidentes por animais peçonhentos registrados pelo SINAN no Estado do Rio de Janeiro no período de 2001 a 2005. Revista Brasileira de Epidemiologia, Rio de Janeiro, v.11, n.1, p.114-127, 2008.

FOX, E. G. P.. Biologia, morfologia, e bioquímica de veneno da formiga lava-pés Solenopsis saevissima Smith (Insecta: Hymenoptera: Formicidae). Tese (Doutorado) Universidade Estadual Paulista, 2010.

FRANK, J. H.; KANAMITSU, K.. Paederus, sensu lato (Coleoptera: Staphylinidae): natural history and medical importance. Journal of medical entomology, v.24, n.2, p.155-191, 1987

FREITAS, M. A.. Serpentes brasileiras. Lauro de Freitas: Malha-de-Sapo, 2003

GUSMÃO, M. A. B.; CREÃO-DUARTE, A. J.. Diversidade e análise faunística de Sphingidae (lepisoptera) em área de brejo e caatinga no Estado da Paraíba, Brasil. Revista Brasileira de Zoologia, v.3, n.21, p.491-498, 2004.

HADDAD JÚNIOR, V.. Identificação de enfermidades agudas causadas por animais e plantas em ambientes rurais e litorâneos: auxílio à prática dermatológica. An Bras Dermatol, v.84, n.4, p.343-348, 2009.

HADDAD JÚNIOR, V.; CARDOSO, J. L. C.; LUPI, O. E.; TYRING, S. K.. Tropical dermatology: Venomous arthropods and human skin. Journal of the American Academy of Dermatology, v.67, n.3, p.331, 2012.

HADDAD JÚNIOR, V.; CARDOSO, J. L. C.. Eurismo e lepidopterismo. In: CARDOSO, J. L. C.. Animais Peçonhentos do Brasil. São Paulo: Sarvier, 2009.

HENDERSON, R. W.; DIXON, J. R.; SOINI, P.. On the seasonal incidence of tropical snakes. Milw. Publ. Mus. Contrib. Biol. Geol., v.17, p.1-15, 1978.

INSTITUTO BUTANTAN. Material Didático. São Paulo: 2007.

KERPEL, S. M.; ZACCA, T.; NOBRE, C. E. B.; JÚNIOR, A. F.; XAVIER, M.; FONSECA, A.. Borboletas do Semiárido: conhecimento atual e contribuições do PPBio. In: BRAVO, F.; 
CALOR, A.. Artrópodes do Semiárido: biodiversidade e conservação. Feira de Santana: Printmídia, 2014.

KESSELRING, J.; EBERT, H.. Relação das borboletas encontradas na Mata da Buraquinho, João Pessoa, Paraíba, Brasil. Revista Nordestina de Biologia, v.2, p.105-118, 1979.

KURY, A. B.; CHAGAS-JR, A.; GIUPPONI, A. P.; GONZÁLEZ, A. P.. Amblypygi, Opiliones, Schizomida, Scorpiones and Chilopoda, Tocantins, Brazil. Check List, v.6, n.4, 2010.

LADEIRA, C. G. P.; MACHADO C.. Epidemiologia dos acidentes com animais peçonhentos na região de Ponte Nova, Minas Gerais, Brasil. Journal Health Npeps, v.2, n.1, p.40-57, 2017.

LEOBAS, G. F.; SEIBERT, C. S.; FEITOS, S. B.. Acidentes por animais peçonhentos no Estado do Tocantins: aspectos clínico-epidemiológicos. Desafios, v.2, n.2, p.269-282, 2016.

LIMA, J. S.; MARTELLI JÚNIOR, H.; MARTELLI, D. R. B.; SILVA, M. S. D.; CARVALHO, S. F. G. D.; CANELA, J. D. R.; BONAN, P. R. F.. Perfil dos acidentes ofídicos no norte do estado de Minas Gerais, Brasil. Revista da Sociedade Brasileira de Medicina Tropical, Uberaba, v.42, n.5, p.561-564, 2009.

LIMA, K. E. C.; VASCONCELOS, S. D.. Acidentes com animais peçonhentos: um estudo etnozoológico com agricultores de Tacaratu, Sertão de Pernambuco. Sitientibus Série Ciências Biológicas, v.6, n.2, p.138-144, 2006.

LISE, F.; COUTINHO, S. E. D.; GARCIA, F. R. M.. Características Clínicas do Araneísmo em Crianças e Adolescentes no Município de Chapecó, Estado de Santa Catarina, Brasil. Acta Scientiarum. Health Sciences, v.28, n.1, 2006

LORENZI, C. C. B.. Estudo Estrutural de mastoparanos isolados de vespas solitárias. Dissertação (Mestrado em Biofísica Molecular) - Universidade Estadual Paulista, São José do Rio Preto, 2002.

LUCAS, S.. Spiders in Brazil: Review Article. Toxicon, v.26, n.9, p.759-772, 1988

MALASPINA, O.. Veneno de formigas: alergia e saúde pública. In: CONGRESSO BRASILEIRO DE ENTOMOLOGIA, 20. Anais. Gramado: 2004

MARQUES, O. A. V.; SAZIMA, I.. Old habits die hard: Mouse handling by a pitviper species on a rodent-free island. Amphibia-Reptilia, v.30, n.3, p.435-438, 2009.

MARQUES, O. A. V.; SAWAYA, R. J.; STENDER-OLIVEIRA, F.; FRANÇA. F. G. R.. Ecolgy of the colubrid snake Pseudablates agassizii in South-Earstern South American. The Herpetological Journal, v.16, n.1, p.37-45, 2006.

MARQUES, O. A.; ETEROVICK, A.; ENDO, W.. Seasonal activity of snakes in the Atlantic Forest in Southeastern Brazil. Amphibia-Reptilia, v.22, n.1, p.103-111, 2001.

MORAIS, R. H. P.. Lepidópteros de importância. in: CARDOSO, J. L. C.. Animais Peçonhentos do Brasil. São Paulo: Sarvier, 2009.

MORTARI M. R; CUNHA, A. O. S; OLIVEIRA, L.; GELFUSO, E. A; VIEIRA, E. B.; SANTOS, W. F.. Comparative toxic effects of the venoms from three wasp species of the genus Polybia (Hymenoptera, Vespidae). J Biol Sci, v.5, n.4, p.449-54, 2005.

NEIVA, A.; PENNA, B.. Viagem científica pelo Norte da Baía, Sudoeste de Pernambuco, Sul do Piauí e de Norte a Sul de Goiás. Mem. do Inst. Oswaldo Cruz, Rio de Janeiro, v.8, n.1, p.74-224, 1916.

NÓBREGA, V. M. D.; ALBUQUERQUE, S. G. E. D.; BARROS, I. M. D. C.; MARQUES, M. D. F. L.. Epidemiologia do Araneísmo de 2005 a 2013 no Centro de Assistência Toxicológica, João Pessoa-PB, Brasil. Revista Brasileira de Ciências da Saúde, v.22, n.1, p.21-26, 2018.

NODARI, F. R.; LEITE, M. L.; NASCIMENTO, E.. Aspectos demográficos, espaciais e temporais dos acidentes escorpiônicos ocorridos na área de abrangência da $3 a$ Regional de Saúde - Ponta Grossa, PR, no período de 2001 a 2004. Publicatio UEPG: Ciências Biológicas e da Saúde, v.12, n.1, 2006

OLIVEIRA, M. E.; MARTINS, M.. When and to find a pitviper: activity patterns and habitat use of the lancehead, Bothrops atrox, in central Amazonia, Brazil. Herpetological Natural History, v.8, n.2, p.101-110, 2001.

OLTROGGE, D. F.. La entnoentomología de algunas categorias de la orden Hymenóptera entre los jicaques. Reuníon de mesa redonda de la sociedad mexicana de antropologia, v.14, p.1-13, 1975.

PARAÍBA. UEPB. CONSUNI. Resolução no 116, de 11 de jun. de 2015. Cria o Centro de Assistência e Informação Toxicológica de Campina Grande - CEATOX, e aprova seu Regimento Interno e dá outras providências. João Pessoa: Diário Oficial do Estado da Paraíba, 2015.

PARISE, E. V.. Vigilância e monitoramento dos acidentes por animais peçonhentos no município de Palmas, Tocantins, Brasil. HYGEIA - Revista Brasileira de Geografia Médica e da Saúde, v.12, n.22, p.72-87, 2016.

PARPINELLI, L.; MARQUES, O. A. V.. Seasonal and daily activity in the pale-headed blindsnake Liotyphlops beui (Serpentes: Anomalepidae) in Southearstern Brazil. south American Journal of herpetology, v.3, n.3, p.207-212, 2008.

PINHO, F. M. O.; OLIVEIRA, E. S.; FALEIROS, F.. Acidente Ofídico no Estado de Goiás. Revista Associação Médica Brasileira, São Paulo, v.50, n.1, p.93-96, 2004.

RODRIGUES, A.; RIBEIRO, J.. Alergia a veneno de himenópteros. Revista de Saúde Amato Lusitano, n.32, p.19-24, 2013.

ROJAS, C. A.; GONÇALVES, M. R.; ALMEIDA-SANTOS, S. M.. Epidemiologia dos acidentes ofídicos na região noroeste do estado de São Paulo, Brasil. Revista Brasileira Saúde e Produção Animal, Salvador, v.8, n.3, p.193-204, 2007.

ROSA, J. A. R. D.. Relatório Epidemiológico: Acidentes por Animais Peçonhentos. Bento Gonçalves: Secretaria Municipal de Saúde, 2015.

SACCARO JÚNIOR, N. L.; MATION, L. F.; SAKOWSKI, P. M.. Impacto do desmatamento sobre a incidência de doenças na Amazônia. Texto para a discussão, v.2142, 2015. 
SALOMAO, M. D. G.; ALBOLEA, A. B. P.; GONÇALVES, E. S.; SANTOS, S. M. A.. Animais peçonhentos no município de Guarulhos, São Paulo, Brasil: incidência de acidentes e circunstâncias com vistas a sua prevenção. Pub. Avulsas Inst. Pau Brasil, v.9, p.77-83, 2005.

SANTOS, J. R. L.; THOMAS, S. E. O.; DORVAL, A.; PASSA, M. C.. A etnoentomologia na comunidade Mata Cavalo de Baixo em Nossa Senhora do Livramento, MT, Brasil.

Biodiversidade, v.14, n.2, 2015.

SCHILEYKO, A. A.. Scolopendromorpha. In: ADIS, J.. Amazonian Arachnida and Myriapoda. Pensoft Publishers, 2002. P.479-500.

SINAN. Sistema de informação de agravo de notificação. Acidentes por animais peçonhentos. 2013.

SOARES, M. R. S.; AZEVEDO, C. S.; MARIA, M.. Escorpionismo em Belo Horizonte, MG: um estudo retrospectivo. Revista da Sociedade Brasileira de Medicina Tropical, v.35, n.4, p.359363, 2002.

SOUZA, M. M.; SILVA, M. A.; SILVA, M. J.; ASSIS, N. G. R.. A capital dos marimbondos-vespas sociais Hymenoptera,
Vespidae do município de Barroso, Minas Gerais. MG Biota, v.1, n.3, p.24-38, 2008.

SOUZA, M. M.; ZANUNCIO, J. C.. Marimbondos-Vespas sociais (Hymenoptera: Vespidae). Viçosa: EdUFV, 2012.

TRAGER, J. C.. A revision of the fire ants, Solenopsis geminate Group (Hymenoptera: Formicidae: Myrmicinae). Journal of the New York Entomological Society, p.141-198, 1991.

TURCI, L. C. B.; ALBUQUERQUE, S.; BERNARDE, P. S.; MIRANDA, D. B.. Uso do hábitat, atividade e comportamento de Bothriopsis bilineatus e de Bothrops atrox (Serpentes: Viperidae) na floresta do Rio Moa, Acre, Brasil. Biota Neotropica, v.9, n.3, 2009.

ULYSSES, M. A.; HANAZAKI, N.; LOPES, B. C.. Percepção e uso dos insetos pelos moradores da comunidade do Ribeirão da Ilha, Santa Catarina, Brasil. Biotemas, v.23, n.3, p.191-202, 2010.

ZERINGÓTA, V.; CASTRO, M. M.; LUCIA, T. M. C. D.; PREZOTO, F.. Nesting of the fire ant Solenopsis saevissima (Hymenoptera: Formicidae) in an urban environment. Fla Entomologist. Florida entomologist, v.97, n.2, p.668-673, 2014.

A CBPC - Companhia Brasileira de Produção Científica (CNPJ: 11.221.422/0001-03) detém os direitos materiais desta publicação. Os direitos referem-se à publicação do trabalho em qualquer parte do mundo, incluindo os direitos às renovações, expansões e disseminações da contribuição, bem como outros direitos subsidiários. Todos os trabalhos publicados eletronicamente poderão posteriormente ser publicados em coletâneas impressas sob coordenação da Cognitionis Publishing, da Companhia Brasileira de Produção Científica e seus parceiros autorizados. Os (as) autores (as) preservam os direitos autorais, mas não têm permissão para a publicação da contribuição em outro meio, impresso ou digital, em português ou em tradução. 